\title{
Pattern of pulmonary vasculitis and major vascular involvement in Hughes-Stovin syndrome (HSS): brief report of eight cases
}

\author{
Yasser Emad ${ }^{1} \cdot$ Yasser Ragab $^{2} \cdot$ Ossama Ibrahim $^{3} \cdot$ Ahmed Saad $^{4} \cdot$ Johannes J. Rasker $^{5}$ (D)
}

Received: 2 October 2019 / Revised: 16 November 2019 / Accepted: 22 November 2019 / Published online: 18 December 2019

(C) The Author(s) 2019

\begin{abstract}
To describe the pattern of pulmonary artery vasculitis and the characteristic computed tomographic pulmonary angiography (CTPA) signs in patients with Hughes-Stovin syndrome (HSS). In a retrospective study, the medical records of eight HSS patients (six men), seen between February 2008 and January 2018, were reviewed regarding history, disease characteristics, laboratory investigations, imaging, and treatments. The mean (SD) age was $37.375 \pm 8.65$ years (range 30-55) and mean (SD) follow-up $30 \pm 41.60$ months (range 9-132). In all patients, routine laboratory investigations and complete coagulation profile were done. In all, CTPA studies were performed as well as and Doppler ultrasound for suspected deep vein thrombosis (DVT). Four patients had a history of thrombophlebitis, and DVT was observed in all, in two cases bilateral. Arterial thromboses involving popliteal, tibial, common iliac, and femoral arteries were observed in one patient. All patients had mild to moderate hemoptysis, and one had massive hemoptysis. None of the patients had a history of recurrent mouth and/or genital ulcers, uveitis, or arthritis. In all patients, CTPA identified bilateral pulmonary artery aneurysms (PAAs) with adherent in situ thrombosis and mural enhancement in all patients. Lobar PA branches were involved in all patients, segmental in six and main PA in five patients. Proper immunomodulators were initiated early, with favorable outcome; none was treated with TNF- $\alpha$ antagonists. HSS is a systemic vasculitis that may affect virtually all major veins and arteries in patients with normal coagulation profile. PAAs, adherent in situ thrombosis, and mural wall enhancement are characteristic CTPA signs. Early treatment with immunomodulators is essential.
\end{abstract}

\section{Key Points}

- Hughes Stevin syndrome (HSS) is a systemic vasculitis that may affect virtually all major veins and arteries in patients. It has a normal coagulation profile.

- Computed tomography (CT) pulmonary angiography is considered to be the most important diagnostic tool to assess the degree and the extent of the characteristic pulmonary artery aneurysms, and in situ thrombosis, and mural wall enhancement.

- It is likely that HSS syndrome is often not recognized and misdiagnosed as deep venous thrombosis (DVT) with pulmonary thromboembolism.

- Early treatment with combined immunomodulators is essential to ensure favorable outcome.

Keywords Hughes-Stovin syndrome (HSS) · Pulmonary artery aneurysms · Pulmonary artery in situ thrombosis · Pulmonary vasculitis · Pulmonary vasculitis in Behçet's disease

Johannes J. Rasker

j.j.rasker@utwente.nl

Yasser Emad

Egypt.yasseremad68@gmail.com

Yasser Ragab

yragab61@hotmail.com

Ossama Ibrahim

ossama.ibrahim@mbht.nhs.uk

Ahmed Saad

medosaad@yahoo.com
Rheumatology Department, Faculty of Medicine, Cairo University, Cairo, Egypt

2 Radiology Department, Faculty of Medicine, Cairo University, Cairo, Egypt

3 Morecambe Bay University Hospitals Lancaster, Lancashire, UK

4 Internal medicine Department, Faculty of Medicine, Cairo University, Cairo, Egypt

5 Faculty of Behavioral, Management and Social Sciences, Department Psychology, Health and Technology, University of Twente, Enschede, The Netherlands 


\section{Introduction}

Hughes-Stovin syndrome (HSS) was first described in 1959 by two British physicians, Drs. John Patterson Hughes and Peter George Ingle Stovin, in two white male patients, presenting with deep venous thrombosis (DVT) and segmental pulmonary artery aneurysms (PAAs) [1]. At first, septic thrombophlebitis was postulated as the underlying etiology, leading to intracardiac infected thrombus that shifted into the pulmonary artery (PA) branches, leading to "mycotic" pulmonary artery aneurysms (PAAs) [2, 3]. In 1964, Kirk and Seal [4] concluded that the involvement of leg veins, venules, and cerebral venous sinus thrombosis (CVST) combined with repeatedly negative blood and bone marrow cultures made a "mycotic" process unlikely. In the following years, the etiology of HSS was ascribed to systemic venous angiitis or collagen disease $[5,6]$. Since then, corticosteroids were generally used as first-line treatment in HSS to control the widespread vascular affection [4-6]. Generally, HSS is considered to result from a vasculitis similar to that seen in Behçet's disease (BD). Some investigators suggest that HSS is actually a subtype of BD, but we can only speculate about that, as the etiology of both diseases is still uncertain. Such a link seems likely as occasionally HSS has been described as a feature in $\mathrm{BD}$ and even as its presenting manifestation [7-9]. BD is a systemic vasculitis which can affect venous and arterial vessels of any size. Fei et al. [10] evaluated the prevalence and characteristics of vascular involvement in BD. In a total of 796 BD patients, vascular involvement was observed in $12.8 \%$ of the patients and more frequently in male patients. Vascular involvement was the initial presentation of the disease in 28 patients with a male to female ratio 3.86:1; venous lesions were more frequently observed than arterial lesions. The authors mentioned that vascular lesions correlated with a high frequency of cardiac involvement and a low incidence of ocular lesions, genital ulcers, and arthritis.

In this brief report, we describe eight cases with HSS and detail their clinical presentations at disease onset, disease characteristics, and CTPA radiological findings with special attention for pulmonary vasculitis patterns. The treatments applied are described.

\section{Patients and methods}

\section{Clinical assessment}

Eight patients with HSS (six men) were retrospectively recruited from Dr. Erfan General Hospital, Jeddah, Saudi Arabia, and from the Rheumatology Department, Faculty of Medicine, Cairo University, between February 2008 and January 2018. Patients' hospital medical records were reviewed regarding past or present history of DVT, recurrent abortions, painful recurrent mouth and/or genital ulcers, or eye involvement. Other data about chest symptoms like dyspnea, chest pain, cough, and hemoptysis severity (mild hemoptysis is $<20 \mathrm{~mL}$, moderate hemoptysis is 20 to $600 \mathrm{~mL}$, and severe or massive $>600 \mathrm{~mL}$ in $24 \mathrm{~h}$ ) as well as constitutional manifestations like fever, anorexia, and weight loss were noted and recorded. Clinical assessment included any symptoms or signs suggestive of systemic lupus or antiphospholipid syndrome like malar rash, alopecia, livedo reticularis, Raynaud's phenomenon, and any skin lesions suggestive of leukocytoclastic vasculitis.

\section{Laboratory investigations}

In all patients, routine laboratory investigations and complete coagulation profile were done including anticardiolipin antibodies, factor $\mathrm{V}$ Leiden mutation, $\beta 2$ glycoprotein I, prothrombin gene mutation, and protein $\mathrm{C}$ and protein $\mathrm{S}$ assays to exclude any predisposing factors for venous and/or arterial thrombosis.

\section{Radiological investigations}

Plain chest X-rays were made, and computed tomography (CT) pulmonary angiography (CTPA) was performed in all patients according to the standardized CTPA protocols as detailed in a previous report [11]. CTPA images were reviewed and interpreted in each lung filed with special emphasis on PAA distributions along the PA branches including the main, lobar, interlobar, and segmental, and subsegmental branches intra-aneurysmal in situ thrombosis, adherence of the thrombus to the pulmonary artery aneurysm (PAA) arterial wall, PAA mural wall enhancement on post-contrast CTPA images, largest diameter of PAA in millimeters, and its distribution were also noted and recorded, and the same was applied for bronchial arteries.

\section{Ethics}

The local ethical committee approved the study design. All patients gave informed written consent to be enrolled into the study according to the Declaration of Helsinki.

\section{Results}

Eight patients with HSS (six men) were retrospectively included in this report. Their mean (SD) age in years was $37.375 \pm 8.65$ ranging between 30 and 55 years. Their mean (SD) disease duration in months was $45.38 \pm 41.15$ and ranged between 15 and 132 months. Their mean duration of follow-up (SD) was $30 \pm 41.60$ ranging between 9 and 132 months. None of the patients had a present or past history 
of recurrent painful mouth and/or genital ulcers, and none had recent or previous attacks of uveitis or arthritis. Four patients had thrombophlebitis, and deep vein thrombosis (DVT) was observed in all patients with bilateral involvement in only two cases, proven by Doppler ultrasound. Iliac veins were affected in two cases, common femoral veins and popliteal veins, each in six patients. Unilateral arterial thrombosis affecting the popliteal, tibial, femoral, and common iliac arteries without aneurysm formation was observed in one patient. All patients had mild to moderate hemoptysis, and one had massive hemoptysis.

Their mean (SD) ESR 1st hour was $45.50 \pm 19.86$ and ranged between 22 and $77 \mathrm{~mm} / \mathrm{h}$, mean CRP was $13.33 \pm$ 14.64 and ranged between 2 and $46 \mathrm{mg} / \mathrm{dL}$, and mean (SD) hemoglobin level was $10.45 \pm 1.703$ and ranged between 8.3$13.1 \mathrm{~g} / \mathrm{dL}$. Four patients had anemia, two had mild leukocytosis, and two patients had thrombocytosis.
All patients showed normal coagulation profile with normal values of anti-cardiolipin antibodies, no factor $\mathrm{V}$ Leiden mutation, $\beta 2$-glycoprotein I, prothrombin gene mutation, and protein $\mathrm{C}$ and protein $\mathrm{S}$ assays.

Detailed demographic, clinical disease characteristics, laboratory findings, and CTPA findings are summarized in Table 1.

Plain chest X-rays were abnormal in seven cases; CTPA showed abnormal findings in all cases (Figs. 1 and 2). All patients showed PAAs and intra-aneurysmal in situ thrombosis (Fig. 1b-d and Fig. 2d) with bilateral lung field involvement in all patients. Lobar PA branches were involved in all patients, segmental in 6 cases, and main PA in five cases (Fig. 2 a, b), while none of the patients showed bronchial artery involvement. The CTPA findings are detailed in Figs. 1 and 2.

The treatment of all patients included pulse IV methylprednisolone therapy in a dose of $1000 \mathrm{mg} /$ day for five

Table 1 Demographic and disease characteristics, laboratory radiological findings, and lines of treatment used in the studied group of patients

\begin{tabular}{|c|c|c|c|c|c|c|c|c|}
\hline Variable & Case 1 & Case 2 & Case 3 & Case 4 & Case 5 & Case 6 & Case 7 & Case 8 \\
\hline Age (years) & 30 & 35 & 32 & 55 & 40 & 44 & 30 & 33 \\
\hline Gender & Male & Male & Male & Male & Female & Female & Male & Male \\
\hline Nationality & Egyptian & Egyptian & Egyptian & Egyptian & Egyptian & Egyptian & Arabian & Egyptian \\
\hline Disease duration (months) & 24 & 36 & 24 & 132 & 84 & 27 & 21 & 15 \\
\hline First presentation & DVT & DVT & Phlebitis & DVT & DVT & DVT & DVT & Hemoptysis \\
\hline Weight loss & Negative & Negative & Positive & Negative & Negative & Negative & Positive & Positive \\
\hline Dyspnea & Positive & Positive & Positive & Positive & Positive & Positive & Positive & Positive \\
\hline Cough & Positive & Positive & Positive & Positive & Positive & Positive & Positive & Positive \\
\hline Fever & Negative & Positive & Positive & Negative & Negative & Positive & Positive & Positive \\
\hline Pleuritic chest pain & Positive & Negative & Negative & Negative & Negative & Negative & Negative & Negative \\
\hline ESR 1st hour (mm/h) & 66 & 30 & 22 & 34 & 44 & 31 & 77 & 60 \\
\hline $\mathrm{CRP}(\mathrm{mg} / \mathrm{dL})$ & 23 & 8.5 & 9.4 & 2.1 & 5.3 & 8.2 & 4.2 & 46 \\
\hline $\mathrm{HB}(\mathrm{gm} / \mathrm{dL})$ & 8.3 & 13.1 & 12 & 11.3 & 10.1 & 9.3 & 11 & 8.5 \\
\hline WBCs $\left(10^{10} / \mathrm{L}\right)$ & 9 & 11.7 & 7.4 & 6.2 & 7.1 & 8.2 & 11.5 & 8 \\
\hline Platelet count $\left(10^{3} / \mu \mathrm{L}\right)$ & 370 & 184 & 230 & 220 & 204 & 350 & 550 & 624 \\
\hline DVT & Positive & Positive & Positive & Positive & Positive & Positive & Positive & Positive \\
\hline Thrombophlebitis & Positive & Positive & Positive & Negative & Positive & Negative & Negative & Negative \\
\hline Hemoptysis & Moderate & Mild & Mild & Mild & Moderate & Mild & Moderate & Massive \\
\hline PAAs and in situ thrombosis & Positive & Positive & Positive & Positive & Positive & Positive & Positive & Positive \\
\hline Main PA & Positive & Negative & Negative & Positive & Positive & Negative & Positive & Positive \\
\hline Lobar & Positive & Positive & Positive & Positive & Positive & Positive & Positive & Positive \\
\hline Interlobar & Negative & Negative & Negative & Negative & Negative & Negative & Positive & Positive \\
\hline Segmental & Negative & Negative & Positive & Positive & Positive & Positive & Positive & Positive \\
\hline Size of largest PAA (mm) & 25 & 14 & 12 & 20 & 24 & 22 & 22 & 30 \\
\hline Bilateral affection & Positive & Positive & Positive & Positive & Positive & Positive & Positive & Positive \\
\hline Major vein thrombosis & Positive & Negative & Negative & Negative & Positive & Negative & Negative & Negative \\
\hline Major arterial thrombosis & Negative & Negative & Positive & Negative & Negative & Negative & Negative & Negative \\
\hline Intra-cardiac thrombus & Negative & Negative & Negative & Negative & Negative & Negative & Right atrium & Negative \\
\hline Pulse methylprednisolone & Positive & Positive & Positive & Positive & Positive & Positive & Positive & Positive \\
\hline Oral CS dose (mg/day) & 40 & 30 & 40 & 20 & 30 & 30 & 30 & 40 \\
\hline Duration of oral CS (months) & 12 & 12 & 12 & 132 & 24 & 15 & 12 & 12 \\
\hline Dose of pulse CP (mg/iv/month) & NR & NR & NR & NR & NR & 750 & 1000 & 1000 \\
\hline Duration of CP (months) & NR & NR & NR & NR & NR & 15 & 12 & 12 \\
\hline Azathioprine dose & 150 & 150 & 150 & 150 & 150 & NR & NR & NR \\
\hline Duration of azathioprine (months) & 12 & 12 & 12 & 132 & 24 & $\mathrm{NR}$ & NR & NR \\
\hline Combination therapy & Positive & Positive & Positive & Positive & Positive & Positive & Positive & Positive \\
\hline Anticoagulation & Received & Received & Received & Received & Received & Received & Received & NR \\
\hline Follow-up (months) & 12 & 12 & 24 & 132 & 24 & 15 & 9 & 12 \\
\hline
\end{tabular}

$C P$ cyclophosphamide, $A Z A$ azathioprine, $C S$ corticosteroids, $N R$ not received; Combination therapy means: maintenance oral CS combined with either AZA or CP 
Fig. 1 a Sagittal reconstruction computed tomography pulmonary angiography (CTPA) showing large defect of the left main pulmonary artery (PA) (white arrows) adherent to the ventral wall of the artery. b Coronal reconstruction CTPA image showing pulmonary artery aneurysm of the left main PA and intra-luminal in situ thrombosis (white arrow). c Axial CTPA image showing left upper lobar PA branch adherent in situ thrombosis (white arrow). d Axial CTPA image showing in situ thrombosis of the left lower lobar PA branch and notice arterial wall mural enhancement (white arrow)

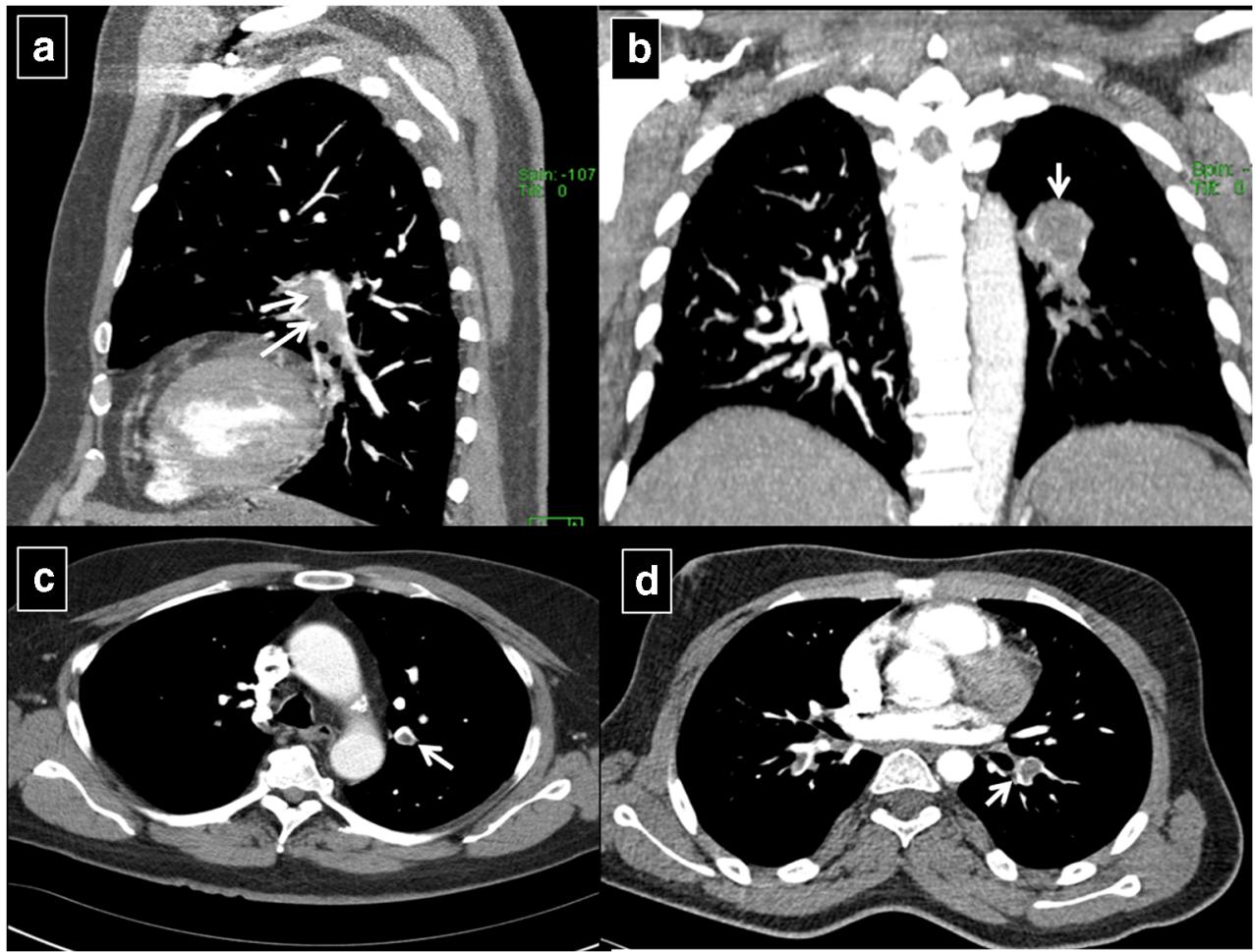

consecutive days as induction therapy; other lines of treatment applied are summarized in Table 1 . None of our patients was treated with TNF- $\alpha$ antagonists or other biologicals. Seven patients had been treated with (short acting) anticoagulants; in one case, this was not feasible with massive hemoptysis. All patients improved regarding hemoptysis frequency and severity, and DVT without any recorded new events compared to the baseline at disease onset and no fatal outcomes were recorded during the duration of their follow-up.

\section{Discussion}

In this report, we describe the pattern of pulmonary vasculitis in a series of patients with HSS. To date, no criteria have been proposed to diagnose HSS; however, it is now established that recurrent thrombophlebitis and/or DVT, PAAs, and intraaneurysmal in situ thrombosis with variable degree of hemoptysis are the constant and key features in patients with HSS otherwise normal coagulation profile [9]. In our report, all patients presented with DVT and other major vein thrombosis. Widespread vascular thrombotic events are dominant features in HSS, e.g., vena cava, intra-cardiac, jugular vein, iliac vein, femoral vein, and portal veins [9], and cerebral venous sinus thrombosis (CVST) [7]. Moreover, extensive arterial thrombosis reaching up to the level of the aorta and common iliac and celiac artery aneurysm was recently reported [9]. In this series, none had ocular lesions, genital ulcers, or arthritis. This is compatible with the findings of Fei et al. who found lower incidence of ocular involvement, genital ulcers, and arthritis in BD patients with vascular lesion than in those without [10].

In our report, all patients showed PAAs in different PA branches and ranging from main PA up to other peripheral branches, e.g. lobar, inter-lobar, and segmental, and distributed in both lung fields, and all PAAs are seats of intraaneurysmal in situ thrombosis and mural wall enhancement on post-contrast CTPA images. An important detail that merits consideration in HSS is that the clots in the PA branches in HSS are mostly due to underlying arterial vasculitis rather than venous thromboembolism; the latter fact was explained recently in one patient with superficial thrombophlebitis without actual DVT who developed PAAs and intra-aneurysmal adherent in situ thrombosis [9]. Moreover, the thrombi in the lower extremities in BD and HSS are tightly adherent to the inflamed veins with no tendency for propagation [11]. Nevertheless, in HSS-related pulmonary vasculitis, intraluminal thrombus evolve in situ due to underlying arterial wall vasculitis, which was documented by Hughes and Stovin themselves on pulmonary autopsy findings in two patients with ruptured PAAs [1]. Important to note in our report is that we showed that intra-aneurysmal in situ thrombosis which is adherent to arterial wall and mural wall enhancement seen on post-CTPA are characteristic and important radiological signs, which are reflecting true arterial wall inflammation due to the underlying vasculitic process; the latter is important to examine in future research involving larger number of patients in this domain. Taken together, pulmonary vasculitis in HSS can activate the coagulation cascade and initiate in situ thrombosis 
Fig. 2 a Coronal reconstruction CTPA image illustrating in situ thrombosis of the right main PA adherent to the mural wall and dilated right main PA. b Coronal reconstruction CTPA image showing bilateral main pulmonary arteries and lower lobar in situ thrombosis (white arrow). c Coronal reconstruction CTPA image showing eccentric in situ arterial thrombosis adherent to the mural arterial wall of the right main PA and lower lobar PA branch (white arrow). d Coronal reconstruction CTPA image showing eccentric in situ thrombosis and mural wall enhancement (white arrow) of the left lower lobar PA branch

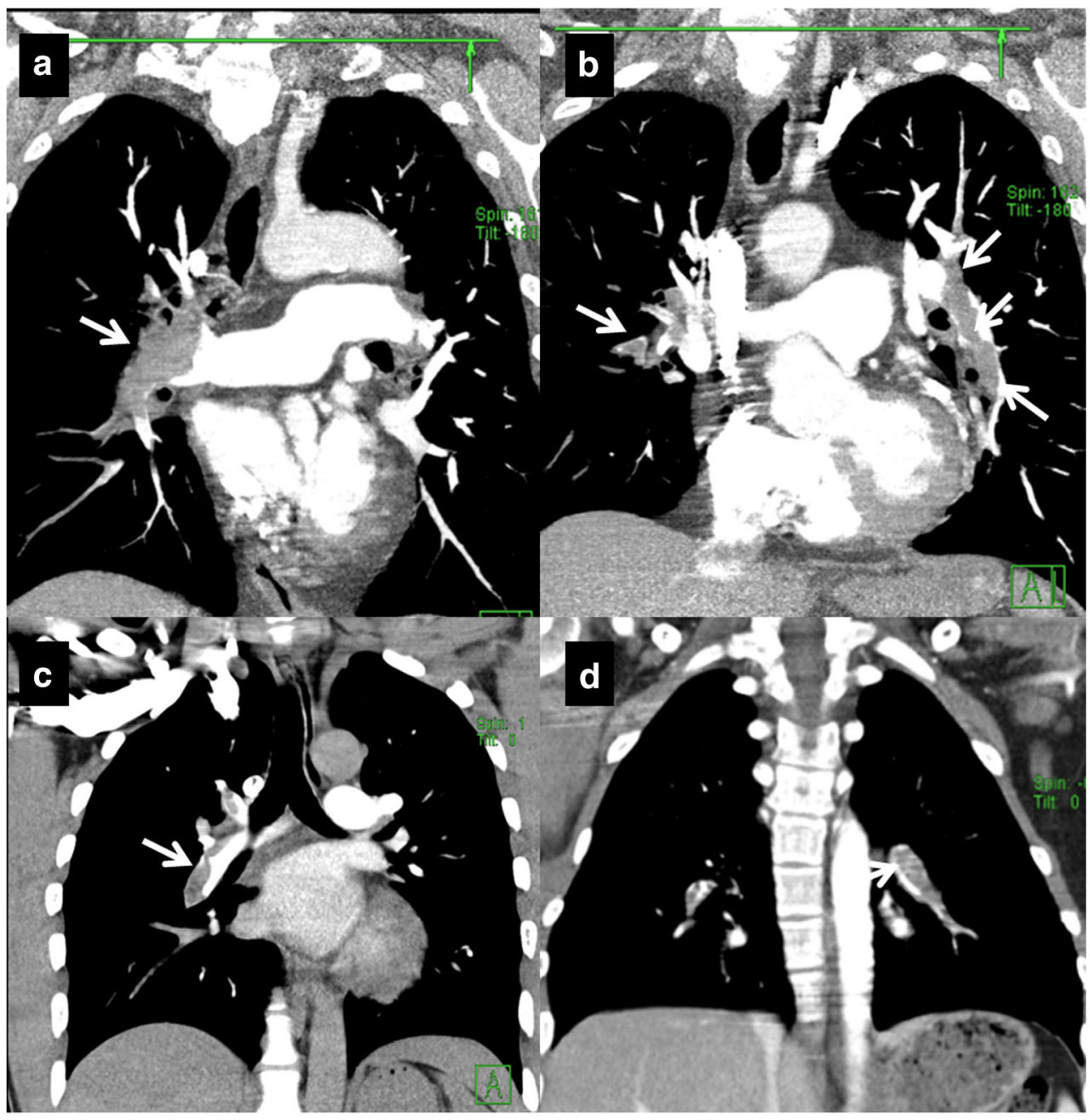

and if left untreated may lead to rupture of PAA, with potential communication to an adjacent bronchus and eventually massive and fatal suffocative hemoptysis, which was recorded as the cause of death and in the autopsy findings in the early report by Hughes and Stovin [1].

In our case series in all patients, we used pulse methylprednisolone as the initial line of treatment in a dose of $1000 \mathrm{mg}$ for five consecutive days as induction therapy; thereafter, oral corticosteroid therapy was prescribed in all patients as illustrated in Table 1. For maintenance of remission, we used, besides oral corticosteroids, either azathioprine in a dose of $150 \mathrm{mg} /$ day (in five patients) or pulse cyclophosphamide for at least 1 year duration (in the other three patients). The treatment of pulmonary vasculitis in HSS and BD generally follows the same lines, as these are the only two conditions known to predispose to PAAs [7, 9, 11].

For the management of BD-related pulmonary vasculitis, the updated EULAR recommendations [12] advise, for treating PAAs, the use of high-dose glucocorticoids and cyclophosphamide and in refractory cases monoclonal anti-TNF antibodies should be considered. If there is a high risk of major bleeding, generally, PA embolization should be preferred to open surgery. Acute DVT in BD patients, and thus also in HSS cases, should be treated with glucocorticoids and immunosuppressives such as azathioprine, cyclophosphamide, or cyclosporine-A. In case of aneurysms of the aorta or in peripheral arteries, medical treatment with cyclophosphamide and corticosteroids is recommended as first choice, before considering operative repair [12]. The recurrence rate of DVT is significantly decreased when these patients are treated with immunosuppressives while anticoagulants did not reduce recurrence of DVT as shown in a meta-analysis of casecontrol studies [13]. If a patient also has extensive acute thrombosis, starting anticoagulation may be considered like in seven of our cases. When there is massive hemoptysis, due to leaking PAA, this is contraindicated, like in one of our patients. The decision to start with anticoagulants should be decided for each case individually, considering the patient presentation and the extent and severity of pulmonary vasculitis. The issue of anticoagulation in patients with HSS (and BD) remains complex, and further studies are needed before definite recommendations can be made. The use of anticoagulants and anti- 
fibrinolytic agents in $\mathrm{BD}$ is not currently recommended by EULAR [12]. As biologic treatments, mostly TNFinhibitors have become more important in the treatment of patients with $\mathrm{BD}$, and further studies are needed to determine the place of biologics in HSS [13].

The strength of our report is that we presented detailed CTPA findings in eight patients with HSS simultaneously and illustrated a comprehensive approach to reach the correct diagnosis at an early stage and to avoid misdiagnosis with pulmonary thromboembolism. Moreover, the CTPA images presented can be used as reference images for the pattern of pulmonary vasculitis in HSS and as a diagnostic tool of assessment in this domain.

\section{Conclusions}

HSS is a systemic vasculitis that can virtually affect all minor or major venous and/or arterial vessels, intracardiac thrombosis, and CVST. In patients presenting with widespread vascular thrombosis, where HSS is clinically suspected, screening of coagulation profile seems mandatory. PAAs and adherent intra-aneurysmal in situ thrombosis and mural wall enhancement on post-contrast images are the main CTPA findings characteristic of pulmonary vasculitis in HSS, the latter can affect all PA branches ranging from the main PA to other peripheral branches, e.g., lobar, interlobar, and segmental. In this clinical setting, CTPA is considered to be the most important diagnostic tool to assess the degree and the extent of PA branches involved first, to establish the diagnosis and to start effective lines of treatment in order to avoid serious and potentially fatal consequences. Further studies are needed to determine the role of biologics in the treatment of HSS syndrome.

Acknowledgments We thank the colleagues at the hospital for their cooperation.

\section{Compliance with ethical standards}

\section{Disclosures None.}

Open Access This article is licensed under a Creative Commons Attribution 4.0 International License, which permits use, sharing, adaptation, distribution and reproduction in any medium or format, as long as you give appropriate credit to the original author(s) and the source, provide a link to the Creative Commons licence, and indicate if changes were made. The images or other third party material in this article are included in the article's Creative Commons licence, unless indicated otherwise in a credit line to the material. If material is not included in the article's
Creative Commons licence and your intended use is not permitted by statutory regulation or exceeds the permitted use, you will need to obtain permission directly from the copyright holder. To view a copy of this licence, visit http://creativecommons.org/licenses/by/4.0/.

\section{References}

1. Hughes JP, Stovin PG (1959) Segmental pulmonary artery aneurysms with peripheral venous thrombosis. Br J Dis Chest 53:19-27

2. Beattie JM, Hall AJ (1912) Multiple embolic aneurysms of pulmonary arteries following thrombosis of veins of leg; death from rupture of aneurysm into lung. Proc R Soc Med 5(Pathol Sect):147-155

3. Pirani CL, Ewart FE Jr, Wilson AL (1949) Thromboendarteritis with multiple mycotic aneurysms of branches of the pulmonary artery. Am J Dis Child 77:460-473

4. Kirk GM, Seal RM (1964) False aneurysm of the pulmonary artery with peripheral venous thrombosis. Thorax 19:449-453

5. Higuchi M, Kitamura S, Terada I (1969) An autopsy case of multiple intrapulmonary artery aneurysms with systemic thromboendophlebitis, the "Hughes-Stovin syndrome". Acta Pathol Jpn 19:69-79

6. Hurlimann Jand Reymond A (1961) [Parietal endocarditis, pulmonary aneurysms, recurring thrombophlebitis]. [Article in French] Ann AnatPathol (Paris). 6:331-46

7. Emad Y, Ragab Y, Shawki A-H, Gheita T, El-Marakbi A, Salama MH (2007) Hughes-Stovin syndrome: is it incomplete Behçet's? Report of two cases and review of the literature. Clin Rheumatol 26:1993-1996

8. Bennji SM, du Preez L, Griffith-Richards S, Smit DP, Rigby J, Koegelenberg CFN et al (2017) Recurrent pulmonary aneurysms: Hughes- Stovin syndrome on the spectrum of Behçet disease. Chest 152:99-103

9. Emad Y, Ragab Y, El-Marakbi A, Saad A, Ibrahim O, Abd-Elhalim A et al (2019) A case of Hughes-Stovin syndrome (incomplete Behçet's disease) with extensive arterial involvement : unmasking the true face of a rare syndrome. Z Rheumatol 78:365-371

10. Fei Y, Li X, Lin S, Song X, Wu Q, Zhu Y, Gao X, Zhang W, Zhao Y, Zeng X, Zhang F (2013) Major vascular involvement in Behçet's disease: a retrospective study of 796 patients. Clin Rheumatol 32: $845-852$

11. Emad Y, Abdel-Razek N, Gheita T, El-Wakd M, El-Gohary T, Samadoni A (2007) Multislice CT pulmonary findings in Behçet's disease (report of 16 cases). Clin Rheumatol 26:879-884

12. Hatemi G, Christensen R, Bang D, Bodaghi B, Celik AF, Fortune F et al (2018) 2018 update of the EULAR recommendations for the management of Behçet's syndrome. Ann Rheum Dis 77:808-818

13. Ozguler Y, Leccese P, Christensen R, Esatoglu SN, Bang D, Bodaghi B, Çelik AF, Fortune F, Gaudric J, Gul A, Kötter I, Mahr A, Moots RJ, Richter J, Saadoun D, Salvarani C, Scuderi F, Sfikakis PP, Siva A, Stanford M, Tugal-Tutkun I, West R, Yurdakul S, Olivieri I, Yazici H, Hatemi G (2018) Management of major organ involvement of Behçet's syndrome: a systematic review for update of the EULAR recommendations. Rheumatology 57:2200-2212

Publisher's note Springer Nature remains neutral with regard to jurisdictional claims in published maps and institutional affiliations. 